\title{
MAX-SLNR Precoding Algorithm for Massive MIMO System
}

\author{
Jing Jiang, Honglin Wang, Yongbin Xie and Wujun Yang \\ Xi'an University of Posts \& Telecommunications, School of Communication and Information Engineering, Xi'an, China
}

\begin{abstract}
Pilot Contamination obviously degrades the system performance of Massive MIMO systems. In this paper, a downlink precoding algorithm based on the Signal-to- Leakage-plus-Noise-Ratio (SLNR) criterion is put forward. First, the impact of Pilot Contamination on SLNR is analyzed, then the precoding matrix is calculated with the eigenvalues decomposition of SLNR, which not only maximize the array gains of the target user, but also minimize the impact of Pilot Contamination and the leak to the users of other cells. Further, a simplified solution is derived, in which the impact of Pilot Contamination can be suppressed only with the large-scale fading coefficients. Simulation results reveal that: in the scenario of the serious pilot contamination, the proposed algorithm can avoid the performance loss caused by the pilot contamination compared with the conventional Massive MIMO precoding algorithm. Thus the proposed algorithm can acquire the perfect performance gains of Massive MIMO system and has better practical value since the large-scale fading coefficients are easy to measure and feedback.
\end{abstract}

\section{Introduction}

At the end of 2010, Thomas 1. Marzetta in Bell Laboratories puts forward the concept of large-scale multiple antennas systems, which is also known as Massive MIMO or Large-Scale MIMO [1], where a large number of antennas are deployed at each cell site. Thanks to large-scale antenna array, cell capacity and spectrum efficiency of users at cell's edge can be improved greatly [2-3] by using gain of array and gain of interference suppression. As antennas' number at base station tends to infinity, the channel capacity tends to infinity according to Information theory. While this theory is not feasible in the application of large-scale MIMO, because obtaining channel state information (CSI) gets harder and pilot contamination in uplink channel estimation becomes inevitable. Pilot contamination had been put forward and analyzed firstly in the literature [4]: large-scale MIMO system usually works in TDD which has channel reciprocity in order to avoid the feedback overhead of CSI, where base station does MIMO precoding in downlink by utilizing uplink channel state information. When users send uplink pilots to base station, pilots of different cells can't guarantee being orthogonal from each other. Non-orthogonal pilots in other cells will produce interference to uplink channel estimation, which makes channel estimation results include not only the CSI from local users to the local base station, but also the contaminated CSI from users in neighboring cells to the local base station. According to this channel estimation results, large-scale MIMO processing can't be accurate, which results in degraded system performance [4] [5]. How to reduce pilot contamination, at present, is becoming a hot spot in academia of large-scale MIMO. The following literatures provide the reference to precoding algorithm which eliminates pilot contamination. How pilot contamination affects the performance of the system is analyzed, and the multi-cell MMSE precoding algorithm had been put forward in literature [6]. In [6], the error between the desired signal and the detected signal in local cell and interference from other cells to local desired users can be minimized at the same time. One kind of multi-layer precoding about large-scale MIMO was put forward in literature [7], the first layer of precoding matrix is used to reduce inter-cell interference and the second precoding matrix is used in the beamforming of sending signals. The third layer of precoding matrix is used for multi-user interference elimination. In the algorithm, channel statistics information is used to reduce the inter-cell interference caused by pilot contamination. Moreover, the implementation of the layer 1 and layer 2 precoding matrix is set in the analog circuits of hardware, and implementation of the third layer is designed in the baseband, so that this kind of composite analog/digital structure reduces complexity of hardware. Similar idea also appears in the literature [8], precoding matrix is divided into inner precoding matrix and outer the precoding matrix, which means intra-cell interference elimination and eliminating inter-cell interference. The 
concept of pilot contamination precoding (PCP) was introduced in literature [9], it mainly says that the base station do the linear combination with slow fading coefficient from terminal to each pilot-reused cell to obtain the slow fading coefficient matrix, then the pilot contamination matrix can be obtained by the product of the slow fading coefficient matrix and conventional single cell linear precoding matrix. It has been proven that the algorithm can eliminate the influence of pilot contamination on precoding based on the SINR of the algorithm and capacity limit. Literature [10] proposed a downlink precoding scheme based on MAX-SINR criteria. Under the condition of keeping base station transmission power constant and making the user's SINR not lower than the setting threshold, it optimizes the weights of precoding to eliminate pilot contamination.

Among the precoding algorithms of MIMO system performance optimization, except the above laws, there is an algorithm based on maximizing Signal-to-Leakage plus-Noise-Ratio(MAX-SLNR). Sadek, Tarighat and Sayed made a perfect expression about classic SLNR precoding algorithm [11]-[12], SLNR precoding can effectively reduce the interference from other users, and maximize the desired signal power of each user, besides, MAX-SLNR allows us substituting generalized eigenvalue problem for the multi-user beamforming.

Based on SLNR and pilot contamination precoding, our algorithm not only optimizes the performance of target users, but also eliminates pilot contamination in the uplink channel estimation and downlink multi-user interference. Compared with multi-cell MMSE algorithm, the algorithm avoids the matrix inversion processing, which makes the complexity of the algorithm reduced greatly. Further, the algorithm takes into account the simplified solution based on slow fading coefficient, so that the pilot contamination can be effectively eliminated under the condition of without the small scale fading information. Due to the large-scale fading information is easy to measure, and feedback cycle is long, of which cost is far less than the small scale fading information feedback, algorithm has more practical application value. Finally, the proposed algorithm can effectively reduce the pilot contamination degradation on system performance through the simulation.

\section{System Model}

In this paper, we consider uplink training and transmit precoding in a multi-cell scenario with $I$ cells in the Massive MIMO TDD system, where each cell consists of a base station with $N_{t}$ antennas and $K$ users with single antenna. Same pilots are reused in $L$ cells among $I$ cells. In uplink training, the signal received at the base station of $\mathrm{j}$-th cell is:

$$
\boldsymbol{y}_{j}=\sum_{l=1}^{L} \sum_{k=1}^{K} \sqrt{p_{r} \tau \beta_{l k}^{j}} \boldsymbol{h}_{l k}^{j} \boldsymbol{x}_{j}+\sum_{i=1, i \neq l}^{I} \sum_{k=1}^{K} \sqrt{p_{r} \tau \beta_{i k}^{j}} \boldsymbol{h}_{i k}^{j} \boldsymbol{x}_{i}+\boldsymbol{n}_{j}
$$

where $\boldsymbol{x}_{j}$ is $\tau$ length pilots which is reused in $L$ cells, $\boldsymbol{x}_{j}$ is the pilot used in $i$-th cell, which is orthogonal with $\boldsymbol{x}_{j} \cdot \beta_{l k}^{j}$ is large-scale fading coefficient between the $k$-th user of $l$-th cell and the base station of $j$-th cell. $\boldsymbol{h}_{l k}^{j}$ is small-scale fading matrix between the $k$-th user of $l$-th cell and the base station of $j$-th cell, elements in matrix are independent and identically distributed (i.i.d.) zeromean, circularly-symmetric complex Gaussian $C N(0,1)$ random variables. Let the pilot power during tansmission at each user be $p_{r}, \quad \boldsymbol{n}_{j}$ is additive noise. By utilizing training sequences ,take the channel estimation with LS algorithm in uplink, the channel state information between the $k$-th user of $j$-th cell and the base station of $j$-th cell can be expressed as:

$$
\hat{\boldsymbol{h}}_{j k}^{j}=\boldsymbol{y}_{j} \cdot \boldsymbol{x}_{j}^{H} / \sqrt{p_{r} \tau}=\sqrt{\beta_{j k}^{j}} \boldsymbol{h}_{j k}^{j}+\sum_{l=1, l \neq j}^{L} \sum_{k=1}^{K} \sqrt{\beta_{l k}^{j}} \boldsymbol{h}_{l k}^{j}+\boldsymbol{n}_{j}^{\prime}(2)
$$

In ( 2 ), the first part $\sqrt{\beta_{j k}^{j}} \boldsymbol{h}_{j k}^{j}$ is the real channel matrix, the second part $\sum_{l=1, l \neq j}^{L} \sum_{k=1}^{K} \sqrt{\beta_{l k}^{j}} \boldsymbol{h}_{l k}^{j}$ is the pilot contamination caused by the cells that use the same training sequences.

In Massive MIMO system, the signal received at the $k$ th user of $j$-th cell is:

$\boldsymbol{y}_{j k}=\sqrt{p_{f}} \boldsymbol{f}_{j k}^{j} \boldsymbol{a}_{j k} \boldsymbol{q}_{j k}+\sum_{i=1, i \neq j}^{I} \sum_{k=1}^{K} \sqrt{p_{f}} \boldsymbol{f}_{j k}^{i} \boldsymbol{a}_{i k} \boldsymbol{q}_{i k}+\boldsymbol{z}_{j k}$

In (3), let transmission power at base station be $p_{f} ; \boldsymbol{q}_{i k}$ is signal transmitted in downlink, which satisfys $E\left[\boldsymbol{q}_{i k} \boldsymbol{q}_{i k}^{H}\right]=\boldsymbol{I} ; \quad \boldsymbol{a}_{j k}$ is downlink precoding vector, which satisfies $\operatorname{tr}\left\{\boldsymbol{a}_{i k}^{H} \boldsymbol{a}_{i k}\right\}=1 ; \boldsymbol{z}_{j k}$ is additive noise at the $k$-th user of $j$-th cell, which satisfies $\boldsymbol{z}_{j k} \in C N(0,1) ; \boldsymbol{f}_{j k}^{j}$ is downlink channel matrix between the $k$-th user of $j$-th cell and the base station of $j$ th cell, first part of ( 3 ) is the desired signal, second part is the interference signal from the base station at adjacent cells.

\section{Precoding Algorithm}

Signal-leakage-noise-ratio (SLNR) is defined as the ratio of the energy of the user's useful signal to noise power and the sum of the energy of user's leakage to other cells. According to the downlink system model (3), the SLNR at the $k$-th user of $j$-th cell can be expressed as: 


$$
S L N R_{j k}=\frac{\left|\boldsymbol{f}_{j k}^{j} \boldsymbol{a}_{j k}\right|^{2}}{\left|\boldsymbol{z}_{j k}\right|^{2}+\left|\sum_{i=1, i \neq j}^{I} \sum_{k=1}^{K} \boldsymbol{f}_{i k}^{j} \boldsymbol{a}_{j k}\right|^{2}}=\frac{\left|\boldsymbol{f}_{j k}^{j} \boldsymbol{a}_{j k}\right|^{2}}{1+\left|\sum_{i=1, i \neq j}^{I} \sum_{k=1}^{K} \boldsymbol{f}_{i k}^{j} \boldsymbol{a}_{j k}\right|^{2}}
$$

During downlink transmission, According to SLNR's eigenvalue decomposition of (4),precoding vector $\boldsymbol{a}_{j k}$ can be obtained at base station. According to channel reciprocity, downlink channel matrix is $\boldsymbol{f}_{j k}^{j}=\left(\sqrt{\beta_{j k}^{j}} \boldsymbol{h}_{j k}^{j}\right)^{T}$.Instead of obtaining the real uplink channel state information directly, the sender get the uplink channel matrix $\hat{\boldsymbol{H}}_{j k}^{j}$ estimated by launch pilot training sequence sent from user to base station through (2). Analysis of (2) shows that the estimated channel matrix contains the true channel state information, and pilot contamination caused by reusing pilot sequences in different cells. Power of the desired signal calculated by the channel estimation is:

$$
\begin{aligned}
& \left|\left(\hat{\boldsymbol{h}}_{j k}^{j}\right)^{T} \boldsymbol{a}_{j k}\right|^{2}=\left|\left(\sqrt{\beta_{j k}^{j}} \boldsymbol{h}_{j k}^{j}+\sum_{l=1, l, \neq j}^{L} \sum_{k=1}^{K} \sqrt{\beta_{l k}^{j}} \boldsymbol{h}_{l k}^{j}+\boldsymbol{n}_{j}^{\prime}\right)^{T} \boldsymbol{a}_{j k}\right|^{2} \\
& =\left|\left(\sqrt{\beta_{j k}^{j}} \boldsymbol{h}_{j k}^{j}\right)^{T} \boldsymbol{a}_{j k}\right|^{2}+\left|\left(\sum_{l=1, l \neq j}^{L} \sum_{k=1}^{K} \sqrt{\beta_{l k}^{j}} \boldsymbol{h}_{l k}^{j}\right)^{T} \boldsymbol{a}_{j k}\right|^{2}+\frac{\boldsymbol{I}}{\sqrt{p_{r} \tau}}
\end{aligned}
$$

For the channel from different users to the base station as $\boldsymbol{h}_{j k}^{j}$ and $\boldsymbol{h}_{j k}^{j}$, and the noise $\boldsymbol{n}^{\prime}$ is independent, so as the antennas' number of base station tends to infinity, cross multiplication of independent vector is 0 according to the law of large numbers, then (5) can be simplified. In the end, the first term of (5) is power of the desired signal, which can be obtained at the sender according to the real channel.The second term is energy of interference caused by pilot contamination in channel estimation. Under the condition of obtaining uplink channel estimation information merely, energy of the pilot contamination must be minimum in order to make the energy gain of desired signal greater. Furthermore, energy of the desired signal calculated according to the channel estimation information can get as close as possible to energy of the useful signal obtained in the real channel. Therefore, when the sender gets channel estimation information merely, influence of pilot contamination in the uplink channel estimation must be considered, and (4) can be derived in further:

$$
S L N R_{j k}=\frac{\left|\hat{\boldsymbol{h}}_{j k}^{j} \boldsymbol{a}_{j k}\right|^{2}}{1+\left|\sum_{l=1, l \neq j}^{L} \sum_{k=1}^{K} \sqrt{\beta_{l k}^{j}} \boldsymbol{h}_{l k}^{j T} \boldsymbol{a}_{j k}\right|^{2}+\left|\sum_{i=1, i \neq j}^{I} \sum_{k=1}^{K} \sqrt{\beta_{i k}^{j}} \boldsymbol{n}_{i k}^{j T} \boldsymbol{a}_{j k}\right|^{2}}
$$

In order to maximize SLNR, downlink precoding vector $\boldsymbol{a}_{j k}$ can be calculated as:

$$
\boldsymbol{a}_{j k}=\arg \max \frac{\left(\hat{\boldsymbol{h}}_{j k}^{j T}\right)^{H} \hat{\boldsymbol{h}}_{j k}^{j T}}{1+\sum_{l=1, l \neq j}^{L} \sum_{k=1}^{K} \beta_{l k}^{j}\left(\boldsymbol{h}_{l k}^{j T}\right)^{H} \boldsymbol{h}_{l k}^{j T}+\sum_{i=1, i \neq j}^{I} \sum_{k=1}^{K} \beta_{i k}^{j}\left(\boldsymbol{h}_{i k}^{j T}\right)^{H} \boldsymbol{h}_{i k}^{j T}}
$$

(7) can be expressed as (8) .

according to (8), When the antenna number of base station tends to infinity, according to the law of large numbers, $\left(\boldsymbol{h}_{l k}^{j \boldsymbol{T}}\right)^{\boldsymbol{H}} \boldsymbol{h}_{l k}^{j \boldsymbol{T}}=I$, then (8) can be expressed as (9).

Therefore precoding vector $\boldsymbol{a}_{j k}$ can be further simplified as the value in relation to large-scale fading in other cells.

$$
\boldsymbol{a}_{j k} \propto \text { max.eigvector }\left[\left(1+\sum_{l=1, l \neq j}^{L} \sum_{k=1}^{K} \beta_{l k}^{j}\left(\boldsymbol{h}_{l k}^{j \boldsymbol{T}}\right)^{\boldsymbol{H}} \boldsymbol{h}_{l k}^{j \boldsymbol{T}}+\sum_{i=1, i \neq j}^{I} \sum_{k=1}^{K} \beta_{i k}^{j}\left(\boldsymbol{h}_{i k}^{j \boldsymbol{T}}\right)^{\boldsymbol{H}} \boldsymbol{h}_{i k}^{j \boldsymbol{T}}\right)^{-1}\left(\left(\hat{\boldsymbol{h}}_{j k}^{j} \boldsymbol{T}\right)^{\boldsymbol{H}} \hat{\boldsymbol{h}}_{j k}^{j \boldsymbol{T}}\right)\right]
$$

$$
\begin{aligned}
& \lim _{N_{t} \rightarrow \infty} \boldsymbol{a}_{j k}=\operatorname{argmax} \frac{\left(\hat{\boldsymbol{h}}_{j k}^{j}\right)^{\boldsymbol{H}} \hat{\boldsymbol{h}}_{j k}^{j \boldsymbol{T}}}{1+N_{t} \sum_{l=1, l \neq j}^{L} \sum_{k=1}^{K} \beta_{l k}^{j} \boldsymbol{I}+N_{t} \sum_{i=1, i \neq j}^{I} \sum_{k=1}^{K} \beta_{i k}^{j} \boldsymbol{I}} \\
& =\operatorname{argmax} \frac{\left(\hat{\boldsymbol{h}}_{j k}^{j} \boldsymbol{T}\right)^{\boldsymbol{H}} \hat{\boldsymbol{h}}_{j k}^{j \boldsymbol{T}}}{\frac{1}{N_{t}}+\sum_{l=1, l \neq j}^{L} \sum_{k=1}^{K} \beta_{l k}^{j} \boldsymbol{I}+\sum_{i=1, i \neq j}^{I} \sum_{k=1}^{K} \beta_{i k}^{j} \boldsymbol{I}}=\operatorname{argmax} \frac{\left(\hat{\boldsymbol{h}}_{j k}^{j} \boldsymbol{T}\right)^{\boldsymbol{H}} \hat{\boldsymbol{h}}_{j k}^{j \boldsymbol{T}}}{\sum_{l=1, l \neq j}^{L} \sum_{k=1}^{K} \beta_{l k}^{j} \boldsymbol{I}+\sum_{i=1, i \neq j}^{I} \sum_{k=1}^{K} \beta_{i k}^{j} \boldsymbol{I}}
\end{aligned}
$$




\section{Numerical Results}

In this section, link-level simulation evaluation based on the proposed MAX-SLNR algorithm has been taken. The proposed algorithm is compared with the conventional Massive MIMO precoding algorithm-matched filter(MF) algorith[2] .The simulation parameters are shown in Table I .

Table 1. parameters of link level Massive MIMO simulation based on MAX-SLNR

\begin{tabular}{|c|c|}
\hline \multicolumn{2}{|c|}{ Simulation Parameters of Massive MIMO } \\
\hline Parameters & Set Value \\
\hline Number of cells & 2 \\
\hline Antenna number of base station & 100 \\
\hline Number of users per cell & 1 \\
\hline Antenna array distance & $0.5 \lambda$ \\
\hline Modulation scheme & QPSK \\
\hline Antenna number of user & 1 \\
\hline Antenna array & uniform linear array \\
\hline $\begin{array}{c}\text { Base station /user transmission } \\
\text { power }\end{array}$ & $20 \mathrm{~dB} / 1 \mathrm{~dB}$ \\
\hline $\begin{array}{c}\text { Large-scale fading coefficient } \\
\text { ( slight pilot contamination ) }\end{array}$ & $\begin{array}{l}\beta_{1 k}^{1}=0.9 \\
\beta_{2 k}^{1}=0.1\end{array}$ \\
\hline $\begin{array}{c}\text { Large-scale fading coefficient } \\
\text { ( severe pilot contamination ) }\end{array}$ & $\beta_{1 k}^{1}=0.5$ \\
$\beta_{2 k}^{1}=0.5$
\end{tabular}

In the uplink, the uplink pilots are configured at terminals in two cells, cell 2 is defined as contamination cell, reusing the same pilot as the cell 1.Firstly, we use the hadamard matrix to generate orthogonal pilot sequences, after QPSK modulation, the original pilot sequences are sent by terminals in different cells, through the corresponding complex gaussian channel. Wireless channel is rapidly changing in reality, assuming that there is only a symbol transmission during a coherent time, satisfying channel reciprocity.

In the downlink, base station in cell1 send data symbols during 1024 different coherence time channel to k-th user in cell 1. After QPSK modulation, the original bitstream start precoding processing for different channel. At this time we compare our MAX-SLNR algorithm with matched filter (MF) precoding algorithms. Downlink MF precoding vector between the $\mathrm{k}$-th user of $\mathrm{j}$-th cell and the base station of $\mathrm{j}$-th cell can be obtained by:

$$
\boldsymbol{a}_{j k}=\left(\hat{\boldsymbol{h}}_{j k}^{j \boldsymbol{T}}\right)^{H} /\left\|\left(\hat{\boldsymbol{h}}_{j k}^{j \boldsymbol{T}}\right)^{H}\right\|
$$

Precoding vector based on MAX-SLNR can be calculated by (9). After going through complex gaussian channel and adding gaussian noise signals, signal is received by MMSE detection. Then, bit error ratio is shown. We draw two kinds of algorithm performance curves with the complete channel information and performance curves only using the large-scale information. The results are as follows:

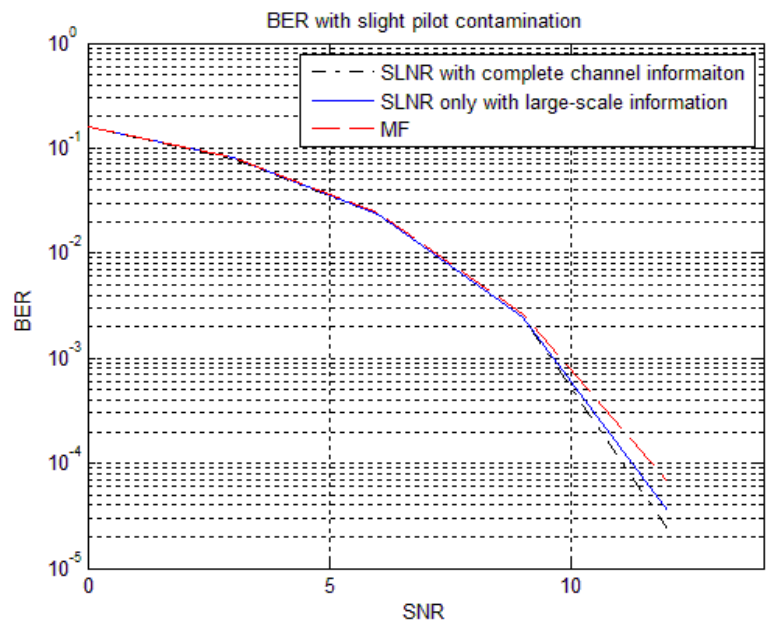

Figure 1（a）

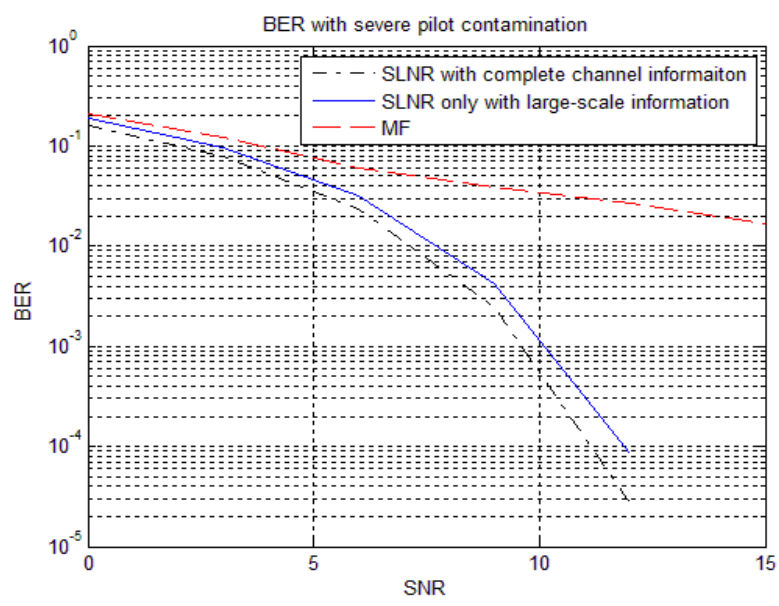

Figure 1 (b)

Figure 1. simulation results of Massive MIMO precoding based on MAX-SLNR

In figure 1 (a), assuming that the large-scale fading coefficients as $\beta_{1 k}^{1}=0.9, \quad \beta_{2 k}^{1}=0.1$. under the condition of slight pilot contamination, as SNR increases, BER curve of the proposed algorithm based on MAX-SLNR is similar to curve of $\mathrm{MF}$ algorithm mentioned in literature[2]. In figure 1 (b) , considering scenario with severe pilot contamination, let the large-scale fading coefficients of two cells be $\beta_{1 k}^{1}=0.5$, $\beta_{2 k}^{1}=0.5$.Under the condition that cell 1 suffers severe pilot contamination from cell 2 , there is an obvious degradation of performance about MF algorithm of large-scale MIMO system. With the increase of SNR, affected by the pilot contamination, imprecise precoding seriously affects the performance of the system, so that BER curve cannot fall. And for MAX-SLNR algorithm based on pilot contamination term and inhibition of interference leakage brought by other cells' users, BER curve decreases obviously with the increase of SNR, the 
performance is much better than the MF algorithm. Compared with slight pilot contamination scenario, there is no obvious degradation of performance with serious pilot contamination. Moreover, the proposed MAXSLNR algorithm only relying on large scale information can obviously inhibit the pilot contamination. The algorithm also has obvious advantage compared with MF algorithm performance.

\section{Conclusions}

The simulation results show that in the case of slight pilot contamination, performance curve of the proposed MAXSLNR algorithm is approximated at that of the conventional Massive MIMO algorithms-MF algorithm. Affected by severe pilot contamination, MAX-SLNR algorithm can effectively reduce the system performance degradation brought by pilot contamination, and reduce the energy of interference leak to adjacent cells, thus gain of large-scale MIMO system performance could be maximized. This paper also puts forward the simplified solution based on the large-scale fading information, which can greatly reduce the channel of information feedback overhead between cells, and simplify the complexity of the channel measurement, which makes the algorithm more practical. In this paper, we assume that the Massive MIMO channel is independent of each other, but large-scale MIMO channel is quite complex in application. We will study the curves of MAX-SLNR performance under the condition of different Channel in the near future.

\section{Acknowledgement}

This paper is sponsored by National 863 Project(2014AA01A705) and the Natural Sience Foundation of ShanXi Province(2014JM8320).

\section{References}

1. T. L. Marzetta, IEEE Trans. Commun., vol. 9, no. 11, pp. 3590-3600, (2010)

2. F. Rusek, D. Persson, B. K. Lau, E. G. Larsson, T. L. Marzetta, O. Edfors, F. Tufvesson, IEEE Signal Process. Mag., vol. 30, no. 1, pp. 40-60, (2012)

3. H. Q. Ngo, E. G. Larsson, T. L. Marzetta, IEEE Trans. Commun., vol. 61, no. 4, pp. 1436-1449, (2012)

4. J. Jose, A. Ashikhmin, T. L. Marzetta, Proc. IEEE International Symposium on Information Theory, pp. 2184-2188,(2009)

5. H. Q. Ngo, E. G. Larsson, T. L. Marzetta, Proc. IEEE International Conference on Acoustics, Speech and Signal Processing, pp. 3464-3467,(2011)

6. J. Jose, A. Ashikhmin, T. L,Marzetta, S. Vishwanath, IEEE Trans. Wireless Commun, vol. 10, no. 8, pp. 2640-2651, (2011)

7. Alkhateeb Ahmed, Leus Geert, W. Heath Jr. Robert, Proc. Asilomar Conference on Signals Systems and Computers, Pacific Grove, CA, Nov. 2014.
8. A. Liu, V. K. N. Lau, IEEE Trans. Signal Process, vol. 62 , no. 18 , pp. 4786-4797,(2014)

9. Li Liangbin, A. Ashikhmin, T. L.Marzetta, Proc of 51st Annual Allerton Conference on Communication, Control, and Computing, pp. 226232.(2013).

10. Jing Jiang, Zheng $\mathrm{Xu}$, International Journal of Future Generation Communication and Networking, vol. 7,no. 3, pp. 107-116,( 2014)

11. A. Tarighat, M. Sadek, A. H. Sayed, Proc of IEEE International Conference on Aoustics, Speech, and Signal Processing, pp. 1129-1132.(2005)

12. M. Sadek, A. Tarighat, A. H.Sayed, IEEE Trans. Wireless Commun., vol. 6, no. 5, pp. 1711-1721, (2007) 